\title{
THE ROLE OF INTERPLANETARY SCATTERING IN WESTERN HEMISPHERE LARGE SOLAR ENERGETIC PARTICLE EVENTS
}

\author{
G. M. Mason, ${ }^{1}$ M. I. Desai,${ }^{2}$ C. M. S. Cohen,${ }^{3}$ R. A Mewaldt,${ }^{3}$ E. C. Stone,${ }^{3}$ and J. R. Dwyer ${ }^{4}$ \\ Received 2006 June 16; accepted 2006 July 6; published 2006 August 1
}

\begin{abstract}
Using high-sensitivity instruments on the $A C E$ spacecraft, we have examined the intensities of $\mathrm{O}$ and $\mathrm{Fe}$ in 14 large solar energetic particle events whose parent activity was in the solar western hemisphere. Sampling the intensities at low $\left(\sim 273 \mathrm{keV}\right.$ nucleon $\left.{ }^{-1}\right)$ and high $\left(\sim 12 \mathrm{MeV}\right.$ nucleon $\left.{ }^{-1}\right)$ energies, we find that at the same kinetic energy per nucleon, the $\mathrm{Fe} / \mathrm{O}$ ratio decreases with time, as has been reported previously. This behavior is seen in more than $70 \%$ of the cases during the rise to maximum intensity and continues in most cases into the decay phase. We find that for most events if we compare the $\mathrm{Fe}$ intensity with the $\mathrm{O}$ intensity at a higher kinetic energy per nucleon, the two time-intensity profiles are strikingly similar. Examining alternate scenarios that could produce this behavior, we conclude that for events showing this behavior the most likely explanation is that the $\mathrm{Fe}$ and $\mathrm{O}$ share similar injection profiles near the Sun, and that scattering in the interplanetary medium dominates the profiles observed at $1 \mathrm{AU}$.
\end{abstract}

Subject headings: acceleration of particles — cosmic rays — interplanetary medium — Sun: abundances — Sun: flares

\section{INTRODUCTION}

When the first detailed observations of heavy elements in solar energetic particle (SEP) events were made in the 1970s, it was discovered that the $\mathrm{Fe}$ intensity reached its maximum before $\mathrm{O}$ at the same kinetic energy per nucleon (O'Gallagher et al. 1976; von Rosenvinge \& Reames 1979; Mason et al. 1983). Later studies of the time to maximum for $\mathrm{Fe}, \mathrm{O}$, and other species, as well as decay time constants, have shown that in many SEP events the differences in temporal behavior are systematically ordered by particle species (Dietrich \& Tylka 2001; Sollitt et al. 2003). The differences in temporal behavior were attributed to the different magnetic rigidity of $\mathrm{Fe}$ versus $\mathrm{O}$, which is due to the partial stripping of ions in SEP events, for example, $(M / Q)_{\mathrm{Fe}}=4.8$ while $(M / Q)_{\mathrm{O}}=2.4$ (Klecker et al. 2000; Möbius et al. 2000).

However, it was not clear which physical mechanisms were responsible for this behavior. For example, Scholer (1976) and Mason et al. (1983) fitted their observations assuming identical injections at the Sun followed by scattering in the interplanetary medium (IPM), including effects of diffusion, convection, and adiabatic deceleration. On the other hand, Mason et al. (1991) showed that two SEP events well fitted at 1 AU using effects of interplanetary propagation could be equally well fitted assuming that the injection at the Sun was extended over a long period followed by nearly scatter-free propagation in the interplanetary medium. Thus, it remains unclear whether the different temporal behavior of these species was due to the acceleration-release process, interplanetary propagation, or some combination of these.

Recent advances in numerical modeling of SEP events associated with coronal mass ejections (CMEs) feature routine inclusion of details of the particle acceleration at the shock followed by interplanetary propagation after particles escape

\footnotetext{
${ }^{1}$ Applied Physics Laboratory, Johns Hopkins University, 11100 Johns Hopkins Road, Laurel, MD 20723; glenn.mason@jhuapl.edu.

${ }^{2}$ Southwest Research Institute, 6220 Culebra Road, San Antonio, TX 78238.

${ }^{3}$ Space Radiation Laboratory, California Institute of Technology, Mail Code 220-47, 1200 East California Boulevard, Pasadena, CA 91125.

${ }^{4}$ Department of Physics and Space Sciences, Florida Institute of Technology, 150 West University Boulevard, Melbourne, FL 32901.
}

the acceleration region. In some cases, the particles undergo significant scattering in the IPM (e.g., $\mathrm{Ng}$ et al. 1999; Li et al. 2005), while in others the transport is nearly scatter-free (Lee 2005). Although solutions from all these models show properties qualitatively similar to the observations, it remains unclear whether the simulated acceleration versus transport effects accurately reflect the processes in large SEP events.

In this Letter, we investigate this issue by examining the temporal behavior of SEP O and Fe over a broad energy range in large SEP events, and we conclude that scattering in the IPM plays a crucial role in a majority of the cases.

\section{OBSERVATIONS}

The observations reported here were carried out with the Ultra Low Energy Isotope Spectrometer (ULEIS; Mason et al. 1998) and the Solar Isotope Spectrometer (SIS; Stone et al. 1998) on the Advanced Composition Explorer (ACE) spacecraft, which was launched into an orbit around the sunward Lagrangian point in 1997. ULEIS is a time-of-flight mass spectrometer covering the energy range $\sim 0.1-10 \mathrm{MeV}$ nucleon $^{-1}$. SIS is a $d E / d x$ versus residual energy spectrometer covering the range $\sim 6-160 \mathrm{MeV}$ nucleon $^{-1}$.

In order to survey typical SEP event properties, we first chose all the events in the NOAA Space Environment Center list of $>10 \mathrm{MeV}$ SEP proton events between the launch of $A C E$ and 2005 January. ${ }^{5}$ All these events were associated with shocks or CMEs. In order to minimize effects arising from magnetic connection, we kept only events with parent activity in the western hemisphere and also discarded events complicated by multiple CMEs (e.g., 1997 November and 2000 July). The resulting group of 14 "simple" SEP events is listed in Table 1.

Figure 1 (left) shows $\mathrm{O}$ and $\mathrm{Fe}$ time-intensity profiles at low $\left(273 \mathrm{keV}\right.$ nucleon $\left.^{-1}\right)$ and high $\left(\sim 12 \mathrm{MeV}\right.$ nucleon $\left.{ }^{-1}\right)$ energies for event 1 . The reasonably prompt rise is typical, but note that at low energies the profile is more complex than a simple rise to maximum and decay (this is typical at low energies). Note also that both $\mathrm{Fe}$ and $\mathrm{O}$ rise at about the same time, and then

\footnotetext{
${ }^{5}$ See http://solar.sec.noaa.gov/ftpdir/indices/SPE.txt.
} 
TABLE 1

14 Western Hemisphere Large SEP Events

\begin{tabular}{|c|c|c|c|c|c|c|c|c|}
\hline \multirow{2}{*}{$\begin{array}{c}\text { EVENT } \\
\text { No. }\end{array}$} & \multirow[b]{2}{*}{ YEAR } & \multicolumn{3}{|c|}{ FLARE } & \multirow{2}{*}{$\begin{array}{c}\text { PEAK } \\
\text { PROTON } \\
\text { FLUX }^{\mathrm{a}}\end{array}$} & \multirow{2}{*}{$\begin{array}{c}\text { EVENT-AVERAGED } \\
\mathrm{Fe} / \mathrm{O}^{\mathrm{b}}\end{array}$} & \multirow{2}{*}{$\begin{array}{c}\text { E-RATio } \\
\text { Low }^{\mathrm{c}}\end{array}$} & \multirow{2}{*}{$\begin{array}{c}E \text {-RAtio } \\
\text { HIGH }^{\mathrm{d}}\end{array}$} \\
\hline & & Maximum & X-Ray Class/Importance & Location & & & & \\
\hline 1 & 1998 & Sep $30,13: 50$ & $\mathrm{M} 2 / 2 \mathrm{~N}$ & N23, W81 & 1200 & $0.195 \pm 0.006$ & 2.0 & 1.9 \\
\hline$\ldots$ & 1999 & Jun 4, 07:03 & $\mathrm{M} 3 / 2 \mathrm{~B}$ & N17, W69 & 64 & $1.063 \pm 0.027$ & 1.4 & 1.0 \\
\hline$\ldots$ & 2000 & Sep $12,12: 13$ & $\mathrm{M} 1 / 2 \mathrm{~N}$ & S17, W09 & 320 & $0.290 \pm 0.007$ & 2.0 & 1.9 \\
\hline$\ldots$ & 2000 & Oct $25,11: 25$ & $\mathrm{M} 2^{\mathrm{e}}$ & $\mathrm{W} 50^{\mathrm{e}}$ & 15 & $0.215 \pm 0.007$ & 1.0 & Stat. $^{\mathrm{f}}$ \\
\hline$\ldots$ & 2001 & Jan $28,16: 00$ & $\mathrm{M} 1 / 1 \mathrm{~N}$ & S04, W59 & 49 & $0.274 \pm 0.007$ & 2.0 & 1.9 \\
\hline$\ldots$ & 2001 & Apr 15, 13:50 & X14/2B & S20, W85 & 951 & $1.325 \pm 0.047$ & 2.0 & 1.4 \\
\hline 7 & 2002 & Apr 21, 01:51 & $\mathrm{X} 1 / 1 \mathrm{~F}$ & S14, W84 & 2520 & $0.752 \pm 0.043$ & 2.8 & 3.9 \\
\hline$\ldots$ & 2002 & Jul 7, 11:43 & M1 & West limb & 22 & $0.028 \pm 0.002$ & 1.4 & Stat. $^{\mathrm{f}}$ \\
\hline$\ldots$ & 2002 & Aug 14, 02:12 & $\mathrm{M} 2 / 1 \mathrm{~N}$ & N09, W54 & 26 & $0.253 \pm 0.009$ & 2.0 & Irr. $^{g}$ \\
\hline $10 \ldots \ldots$ & 2002 & Aug 24, 01:12 & $\mathrm{X} 3 / 1 \mathrm{~F}$ & S08, W90 & 317 & $0.536 \pm 0.014$ & Irr. $^{\mathrm{g}}$ & 1.9 \\
\hline $11 \ldots \ldots$ & 2003 & Nov 4, 19:29 & X28/3B & S19, W83 & 353 & $1.217 \pm 0.030$ & 2.8 & 1.0 \\
\hline $12 \ldots \ldots$ & 2003 & Dec 2, 09:48 & C7 & West limb & 86 & $0.887 \pm 0.022$ & 2.0 & Stat. $^{\mathrm{f}}$ \\
\hline $13 \ldots \ldots$ & 2004 & Apr 11, 04:19 & $\mathrm{C} 9 / 1 \mathrm{~F}$ & S14, W47 & 35 & $0.316 \pm 0.010$ & 2.0 & 1.9 \\
\hline $14^{\mathrm{h}} \ldots \ldots$ & 2005 & Jan 20, 07:01 & X7.9 & N14, W61 & 1860 & $0.261 \pm 0.021$ & 2.8 & 1.9 \\
\hline
\end{tabular}

${ }^{a}$ In units of particles $\mathrm{s}^{-1} \mathrm{~cm}^{-2} \mathrm{sr}^{-1}$ above $10 \mathrm{MeV}$.

${ }^{b}$ At $0.32-0.45 \mathrm{MeV}$ nucleon ${ }^{-1}$ (Desai et al. 2006).

${ }^{\mathrm{c}}$ Ratio of $\mathrm{O}$ kinetic energy per nucleon to $\mathrm{Fe}$ at $276 \mathrm{keV}$ nucleon ${ }^{-1}$ to give a time-invariant ratio.

${ }^{\mathrm{d}}$ Ratio of $\mathrm{O}$ kinetic energy per nucleon to $\mathrm{Fe}$ at $13.2 \mathrm{MeV}$ nucleon ${ }^{-1}$ to give a time-invariant ratio.

${ }^{\text {e }}$ Flare identification from Kahler (2005).

${ }^{\mathrm{f}}$ The statistical accuracy is insufficient to compare intensities accurately.

${ }^{g}$ Irregular temporal variation of the Fe/O ratio precluded finding the energy (if any) at which Fe/O was time-invariant.

${ }^{\mathrm{h}}$ Last in a sequence of three events that occurred between 2005 January 16 and January 20.

$\mathrm{O}$ continues to rise even after the Fe has begun to decay. This behavior produces the decreasing $\mathrm{Fe} / \mathrm{O}$ ratio mentioned earlier.

The right panel of Figure 1 shows the same Fe time-intensity profiles, with $\mathrm{O}$ profiles taken at a higher kinetic energy per nucleon (the ratios of the higher $\mathrm{O}$ energies to $\mathrm{Fe}$ selected for each event are given in the rightmost columns of Table 1). The $\mathrm{O}$ profiles are strikingly similar to the corresponding Fe profiles. This is especially surprising during the rise phase for the low-energy data, since the $546 \mathrm{keV}$ nucleon ${ }^{-1} \mathrm{O}$ has a scatterfree travel time to $1 \mathrm{AU}$ about $2 \mathrm{hr}$ shorter than the $273 \mathrm{keV}$ nucleon $^{-1} \mathrm{Fe}$, a difference that would be easily visible in the figure. (For the high-energy data, the difference in travel times is only $\sim 15$ minutes and so would not be visible.) Note that the similarity of the $\mathrm{Fe}$ intensity to the $\mathrm{O}$ intensity goes beyond a general similarity and holds even for some of the irregular features of the profiles.
Since particle intensities increase rapidly during the rise portion of these events, it is hard to tell whether the similarity between the rise-phase $\mathrm{Fe}$ and $\mathrm{O}$ is greater in the left versus the right panel of Figure 1. However, the ratio of Fe to $\mathrm{O}$ is a more sensitive indicator of the relative behavior of the two species. Figure $2 a$ shows the Fe/O ratios for event 1 , with the left panel showing the ratios at the same energy per nucleon (as in Fig. 1, left) and the right panel showing the ratios with higher energy per nucleon $\mathrm{O}$ (as in Fig. 1, right). The large filled circles mark the time of maximum of the Fe intensity. Note that at the same energy per nucleon the $\mathrm{Fe} / \mathrm{O}$ ratio decreases by an order of magnitude, changing fairly smoothly from the onset and continuing through and well beyond the time of maximum. For the ratio of Fe with higher energy $\mathrm{O}$ (Fig. 2a, right), the systematic decrease of the ratio is gone, and only fluctuations around a nearly constant value remain.
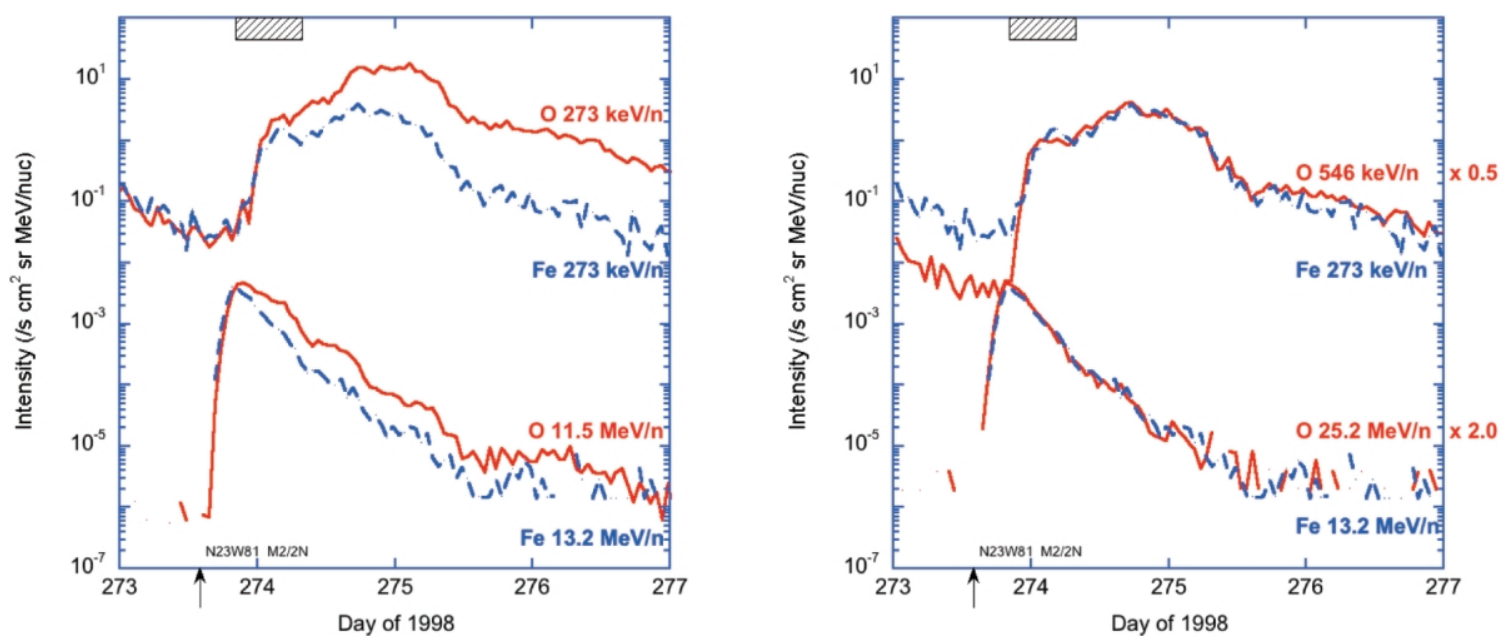

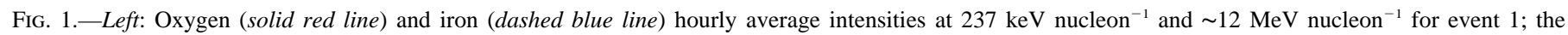

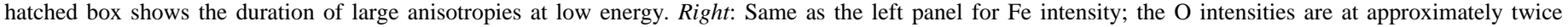
the kinetic energy per nucleon as the left panel and renormalized as shown to facilitate comparison with Fe intensity. 

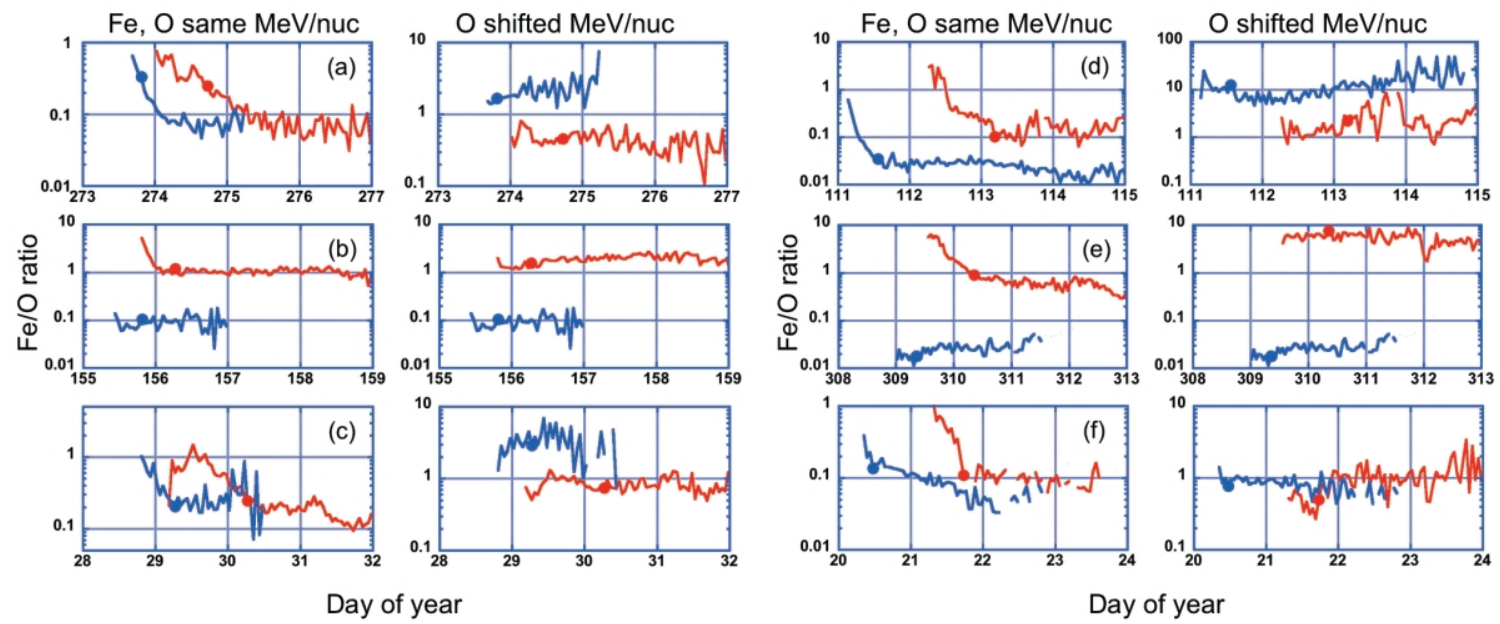

FIG. 2.-Left panels: Fe/O ratio at $273 \mathrm{keV}$ nucleon $^{-1}($ red $)$ and $\sim 12 \mathrm{MeV}$ nucleon ${ }^{-1}$ (blue), for $(a)$ event $1,(b)$ event $2,(c)$ event 5 , $(d)$ event $7,(e)$ event 11 , and $(f)$ event 14. Right panels: The same events, $\mathrm{Fe} / \mathrm{O}$ ratio with $\mathrm{Fe}$ at same energy as before, and $\mathrm{O}$ at a higher energy given by the factor shown in Table 1. Large filled circles mark the time of maximum intensity of Fe. Late times of maximum at $273 \mathrm{keV}$ nucleon ${ }^{-1}$ for some events (e.g., $\left.[c],[d],[f]\right)$ are due to intensity peaking at shock passage.

This is a reflection of the level of agreement between the timeintensity profiles in the right panel of Figure 1.

Figures $2 b-2 f$ show representative pairs of $\mathrm{Fe} / \mathrm{O}$ ratios for other events in Table 1 . Note that in most cases a large systematic decrease in the $\mathrm{Fe} / \mathrm{O}$ ratio is replaced by a nearly constant $\mathrm{Fe} / \mathrm{O}$ ratio when higher energy $\mathrm{O}$ is used. These nearly constant $\mathrm{Fe} / \mathrm{O}$ ratios show that for these events, the Fe intensity profiles are similar to higher energy $\mathrm{O}$ profiles as in Figure 2 $(r i g h t)$. In selecting higher energy $\mathrm{O}$ intensities to compare with $\mathrm{Fe}$, we used standard energy bins that are spaced by a factor of 1.4 on ULEIS and $~ 1.9$ on SIS. There are some other features of these panels that we note here:

1. In several cases (Figs. $2 b, 2 d, 2 e, 2 f$, left) at the same energy per nucleon, the $\mathrm{Fe} / \mathrm{O}$ ratio decrease continues only to the time of maximum and afterward is nearly constant.

2 . In the case of Figures $2 b$ and $2 e$ (left), the lower energy data show a decrease in $\mathrm{Fe} / \mathrm{O}$, while the $\sim 12 \mathrm{MeV}$ nucleon $^{-1}$ ratio is constant at the same $\mathrm{Fe}$ and $\mathrm{O}$ energies (denoted by an $E$-ratio of 1.0 in Table 1 and a repeat of the same energy $\mathrm{Fe} /$ $\mathrm{O}$ ratio in the right panels of Fig. 2).

3. The scatter is larger in the plots with higher energy $\mathrm{O}$, mainly because of poorer counting statistics.

\section{DISCUSSION}

Twelve of the 14 events (see Table 1) show a reasonably flat $\mathrm{Fe} / \mathrm{O}$ ratio after comparing the $276 \mathrm{keV}$ nucleon ${ }^{-1} \mathrm{Fe}$ with higher energy $\mathrm{O}$, and eight of 11 events show such flattening for the $13 \mathrm{MeV}$ nucleon ${ }^{-1} \mathrm{Fe}$ compared with higher energy $\mathrm{O}$. (Three events, Nos. 4, 8, and 12, had poor statistics at high energies, precluding a comparison.) Intermediate-energy comparisons fit smoothly into the pattern shown here, making these properties typical of large western hemisphere SEP events.

How do these features fit into the alternate accelerationrelease versus propagation-dominated scenarios mentioned earlier? For the propagation-dominated case, we follow the recent discussion of Cohen et al. (2005 and references therein). If the release profiles at the source for $\mathrm{Fe}$ and $\mathrm{O}$ are similar, then for a given set of interplanetary conditions the intensities at $1 \mathrm{AU}$ will be controlled by the diffusion coefficient: $\kappa=v \lambda / 3$, where $v$ is the particle speed and $\lambda$ is the scattering mean free path (mfp). Typically, the mfp is a function of particle's magnetic rigidity, so $\lambda \propto(v M / Q)^{\alpha}$, where $\alpha$ is related to the interplanetary turbulence power spectrum. The similarity of the Fe and $O$ profiles implies that their diffusion coefficients are the same, $\kappa_{\mathrm{O}}=\kappa_{\mathrm{Fe}}$, so substituting for $\lambda$ above we deduce the ratio of kinetic energies per nucleon where this condition is met:

$$
E_{\mathrm{O}} / E_{\mathrm{Fe}}=\left[(Q / M)_{\mathrm{O}} /(Q / M)_{\mathrm{Fe}}\right]^{2 \alpha /(\alpha+1)} .
$$

For the typical $(M / Q)$-values quoted above, the quantity in square brackets is 2.052 , so we have

$$
E_{\mathrm{O}} / E_{\mathrm{Fe}}=2.052^{2 \alpha /(\alpha+1)} .
$$

Taking a simple average of the energy ratio values in Table 1, this implies $\alpha=0.9 \pm 0.2$, comparable to recent theoretical estimates (e.g., Shalchi et al. 2004 and references therein). At tens of $\mathrm{MeV}$ per nucleon, the charge state of $\mathrm{Fe}$ is often higher than at low energies; for example, if we use high-energy $\mathrm{O}$ and $\mathrm{Fe}$ ionization states from Leske et al. (1995) or Cohen et al. (1999), the ratio $E_{\mathrm{O}} / E_{\mathrm{Fe}} \sim 1.4-1.6$, similar to some cases listed in Table 1 . The exponent $\alpha$ may also vary from event to event, or even with energy per nucleon, so other factors could contribute to the different energy ratios in Table 1.

For the scenario of acceleration-release followed by nearly scatter-free propagation, the decrease of the $\mathrm{Fe} / \mathrm{O}$ ratio at the same energy per nucleon would be due to the earlier escape of $\mathrm{Fe}$ from the scattering region near the shock. This occurs because its larger $M / Q$ ratio gives $\mathrm{Fe}$ a larger scattering $\mathrm{mfp}$, so it can reach the shock's upstream escape "boundary" more easily than $\mathrm{O}$. The near-equality of the Fe versus higher energy $\mathrm{O}$ time-intensity profiles, however, is a coincidence in this scenario, since after escaping from the shock acceleration region $\mathrm{O}$ would propagate to $1 \mathrm{AU}$ faster than the Fe. For example, the equality we see between $\mathrm{Fe}$ and $\mathrm{O}$ at 273 versus $546 \mathrm{keV}$ nucleon $^{-1}$ requires that the Fe release was $2 \mathrm{hr}$ ahead of the $\mathrm{O}$ release, such that the two particle populations just crossed each other as they reached 1 AU. Similar matching would be required at other energies, for example, a 15 minute 
difference at $10 \mathrm{MeV}$ nucleon $^{-1}$. While this cannot be ruled out for any single event, it seems very unlikely to us that such a coincidence occurs in such a large fraction of the cases. We conclude that for events showing time-invariant $\mathrm{Fe} / \mathrm{O}$ ratios for $\mathrm{O}$ with higher energy per nucleon than $\mathrm{Fe}$, the most reasonable explanation is that the $\mathrm{Fe}$ and $\mathrm{O}$ have similar release profiles near the Sun followed by substantial scattering on the way to $1 \mathrm{AU}$.

Some of the events (e.g., No. 11) occurred during periods of high activity when turbulence could easily have been produced by CMEs, thus explaining the key role of scattering in the IPM. Others, however, occurred when there was little or no other obvious activity. Many of the events studied here are of a type in which electrons arrive promptly (Krucker \& Lin 2000; Haggerty \& Roelof 2002); also regarding the ions, the onset of event 14 was extremely fast from SIS energies to relativistic energies (see, e.g., Bieber et al. 2005). So how is it possible that interplanetary scattering plays such a key role in most of the MeV heavy-ion events studied here? In cases where there is no obvious source of turbulence, we note that all these events had elevated $>10 \mathrm{MeV}$ proton intensities, and that a possible explanation lies in the generation of protonamplified waves generated by the streaming SEPs ( $\mathrm{Ng}$ et al. 1999). The growth rate timescale for these waves is roughly an hour, so high-energy protons and near-relativistic electrons will begin to arrive at $1 \mathrm{AU}$ before the waves have been amplified significantly, allowing prompt arrivals for electrons and high-energy protons and scattering-dominated profiles for the $\mathrm{MeV}$ ions.

Finally, it should be emphasized that the evidence for a critical role for interplanetary scattering presented here is not affected by whether the acceleration-release near the Sun is prompt or delayed; the only source requirement is that the $\mathrm{Fe}$ and $\mathrm{O}$ particle spectra at release are similar.

This work was supported by NASA grant NNG04GJ51G at the Johns Hopkins University Applied Physics Laboratory, NASA grant NAG 5-6912 and NSF grant ATM 04-54428 at the California Institute of Technology, and NASA grant NNG05GQ96G and NSF grant ATM 05-55878 at SwRI.

\section{REFERENCES}

Bieber, J. W., et al. 2005, Proc. 29th Int. Cosmic Ray Conf. (Pune), 1, 237

Cohen, C. M. S., et al. 1999, Geophys. Res. Lett., 26, 149

. 2005, J. Geophys. Res., 110, A09S16, doi: 10.1029/2005JA011004

Desai, M. I., Mason, G. M., Gold, R. E., Krimigis, S. M., Cohen, C. M. S., Mewaldt, R. A., Mazur, J. E., \& Dwyer, J. R. 2006, ApJ, in press

Dietrich, W. F., \& Tylka, A. J. 2001, Proc. 27th Int. Cosmic Ray Conf. (Hamburg), 27, 3173

Haggerty, D. K., \& Roelof, E. C. 2002, ApJ, 579, 841

Kahler, S. 2005, ApJ, 628, 1014

Klecker, B., et al. 2000, in AIP Conf. Proc. 528, Acceleration and Transport of Energetic Particles Observed in the Heliosphere, ed. R. A. Mewaldt et al. (Melville, NY: AIP), 135

Krucker, S., \& Lin, R. P. 2000, ApJ, 542, L61

Lee, M. A. 2005, ApJS, 158, 38

Leske, R. A., Cummings, J. R., Mewaldt, R. A., Stone, E. C., \& von Rosenvinge, T. T. 1995, ApJ, 452, L149

Li, G., Zank, G. P., \& Rice, W. K. M. 2005, J. Geophys. Res., 110, A06104, doi: $10.1029 / 2004 \mathrm{JA} 010600$
Mason, G. M., Gloeckler, G., \& Hovestadt, D. 1983, ApJ, 267, 844

Mason, G. M., Reames, D. V., \& Ng, C. K. 1991, Proc. 22nd Int. Cosmic Ray Conf. (Dublin), 3, 197

Mason, G. M., et al. 1998, Space Sci. Rev., 86, 409

Möbius, E., et al. 2000, in AIP Conf. Proc. 528, Acceleration and Transport of Energetic Particles Observed in the Heliosphere, ed. R. A. Mewaldt et al. (Melville, NY: AIP), 131

Ng, C. K., Reames, D. V., \& Tylka, A. J. 1999, Geophys. Res. Lett., 26, 2145

O'Gallagher, J. J., Hovestadt, D., Klecker, B., Gloeckler, G., \& Fan, C. Y. 1976, ApJ, 209, L97

Scholer, M. 1976, ApJ, 209, L101

Shalchi, A., Bieber, J. W., Matthaeus, W. H., \& Qin, G. 2004, ApJ, 616, 617

Sollitt, L. S., Stone, E. C., Mewaldt, R. A., Cohen, C. M. S., Leske, R. A., Widenbeck, M. E., \& von Rosenvinge, T. T. 2003, Proc. 28th Int. Cosmic Ray Conf. (Tsukuba), 6, 3295

Stone, E. C., et al. 1998, Space Sci. Rev., 86, 357

von Rosenvinge, T. T., \& Reames, D. V. 1979, Proc. 16th Int. Cosmic Ray Conf. (Kyoto), 5, 68 